\title{
Hipotiroidismo subclínico, depresión y deterioro cognitivo: experiencia en un centro de adultos mayores de Lambayeque
}

\author{
Subclinical hypothyroidism, depression and cognitive impairment: experience at a
}

Lambayeque elderly center

\author{
Carol Gonzales-Gonzales ${ }^{1, a}$, Fátima Deza Becerra, ${ }^{1, b}$, Franco León Jiménez ${ }^{1, c}$, \\ Jaquelyn Poma Ortiz ${ }^{1, d}$ \\ Escuela de Medicina, Universidad Santo Toribio de Mogrovejo, Chiclayo, Perú.

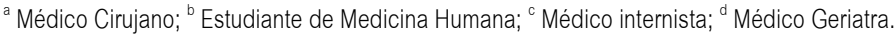

\begin{abstract}
Resumen
Introducción: El hipotiroidismo subclínico en adultos mayores es una enfermedad poco estudiada. Objetivos: Determinar la frecuencia de hipotiroidismo subclínico, depresión y deterioro cognitivo y explorar su asociación, en adultos mayores. Diseño: Estudio descriptivo, transversal; muestreo no probabilístico, consecutivo. Escenario: Centro de adultos mayores de EsSalud, Lambayeque, Perú. Métodos: En 2012 y 2014, y en una muestra de 84 pacientes, se cuantificó TSH y T4L mediante técnica ELISA. Se evaluó depresión con el test de Yesavage reducido y el déficit cognitivo con el mini mental test examination. Se determinó frecuencias, porcentajes y análisis inferencial exploratorio mediante el cálculo de razones de prevalencia, con intervalos de confianza al 95\%, usando Epidat v 3.1 . Principales medidas de resultados: Hipotiroidismo subclínico, depresión y deterioro cognitivo y su asociación. Resultados: Se estudió 65 mujeres (77\%) y 19 hombres (23\%); en 11 (13,1\%) adultos mayores se halló hipotiroidismo subclínico, en $36(42,9 \%)$ depresión y en $11(13,1 \%)$ deterioro cognitivo. No se encontró asociación entre hipotiroidismo subclínico y depresión (RP: 0,85, IC95\% 0,39 a 1,85; p 0,677) o deterioro cognitivo (RP: 2,48, IC $95 \%$ 0,77 a 7,98; p 0,1349). Conclusiones: Existió una elevada frecuencia de hipotiroidismo subclínico, depresión, y deterioro cognitivo en los adultos mayores estudiados. No se evidenció asociación de hipotiroidismo subclínico con depresión o con deterioro cognitivo.
\end{abstract}

Palabras clave: Hipotiroidismo, depresión, deterioro cognitivo leve.

\begin{abstract}
Introduction: There are few studies on subclinical hypothyroidism in the elderly. Objectives: To determine the frequency of subclinical hypothyroidism, depression and cognitive impairment, and explore their association in elderly patients. Design: Descriptive, crosssectional study. Scenario: EsSalud seniors' center, Lambayeque, Peru. Methods: In 2012 and 2014 TSH and T4L were determined by ELISA in 84 elderly patients. Depression was assessed through a reduced Yesavage test and cognitive impairment by the mini mental test. Frequencies and percentages were determined and prevalence ratios with $95 \%$ confidence intervals were estimated using Epidat v3.1. Main outcome measures: Subclinical hypothyroidism, depression and cognitive impairment and their association. Results: Sixtyfive women (77\%) and 19 men (23\%) were included. Subclinical hypothyroidism was found in 11 older adults (13.1\%), depression in 36 $(42.9 \%)$ and cognitive impairment in $11(13.1 \%)$. No association between subclinical hypothyroidism and depression (PR: 0.85, Cl95\% [0.39-1.85], p: 0.677) or subclinical hypothyroidism and cognitive impairment (RP: 2.48, Cl95\% [0.77-7.98], p: 0.1349) were found. Conclusions: There was a high frequency of subclinical hypothyroidism, depression, and cognitive impairment in the elderlies studied. There was no association between subclinical hypothyroidism and depression or cognitive impairment.
\end{abstract}

Keywords: Hypothyroidism, depression, mild cognitive impairment.

An Fac med. 2014;75(4):327-30 / doi: http://dx.doi.org/doi:10.15381/anales.v75i4.10849

\section{INTRODUCCIÓN}

El hipotiroidismo subclínico se define como la presencia de concentraciones séricas de tirotropina (TSH) por encima del valor normal, concentraciones normales de hormonas tiroideas (T3 y T4 libre) y ausencia de manifestaciones clínicas ${ }^{(1)}$. Según la Encuesta Nacional de Salud y Nutrición de Estados Unidos, de 2002, la prevalencia de hipotiroidismo fue $0,3 \%$ y de hipotiroidismo subclínico 4,3\% (2). Martínez, en Paraguay, halló una prevalencia de 1,89\% en la población general ${ }^{(3)}$.
Este trastorno es frecuente en adultos mayores, considerados según OMS como personas con edad mayor o igual a 60 años ${ }^{(4)}$. El cuadro de hipotiroidismo subclínico es inespecífico, pero su presencia está asociada a mayor morbilidad por enfermedad coronaria ${ }^{(5)}$, mayor riesgo de desarrollar insuficiencia cardiaca congestiva, con valores de TSH de 10 a 19,9 mIU/L (hazard ratio: 1,86 , IC95\% 1,27 a 2,72), y posiblemente a aumentos en la mortalidad ${ }^{(6)}$.

Los métodos de cuarta generación utilizan ensayos inmunométricos ca- paces de detectar concentraciones de TSH hasta de $0,001 \mathrm{mUI} / \mathrm{L}^{(2)}$.

Existen múltiples estudios para concertar la conveniencia o no de realizar un cribado hormonal para determinar la prevalencia de hipotiroidismo subclínico, principalmente en la salud mental del adulto mayor ${ }^{(7,8)}$. La depresión es un trastorno mental frecuente, que se caracteriza por la presencia de tristeza, perdida de interés o placer, sentimiento de culpa o falta de autoestima, trastornos del sueño o del apetito, sensación de cansancio y falta de concentración ${ }^{(9)}$. 
Según la OMS, afecta a 121 millones de personas en el mundo y es responsable de unas 850 mil muertes cada año, principalmente por suicidio ${ }^{(10)}$. El deterioro cognitivo es el déficit de las funciones mentales; el más común es la queja de pérdida de la memoria ${ }^{(11)}$. Uno de los cambios más reconocidos que suceden con el envejecimiento es el enlentecimiento de los procesos mentales. La mayor parte de los estudios coinciden en que la prevalencia de la demencia se incrementa al doble cada 5 años a partir de los 60 años de edad, comenzando desde $10 \%$ y con un pico en los mayores de 85 años de hasta $45 \%{ }^{(11)}$.

No se ha encontrado estudios locales en Lambayeque acerca de hipotiroidismo subclínico, su frecuencia ni su asociación con depresión y deterioro cognitivo. Por esto, el objetivo del estudio fue determinar la frecuencia de hipotiroidismo subclínico, depresión y deterioro cognitivo, y explorar su asociación en un centro de adultos mayores de Lambayeque.

\section{MÉTODOS}

Estudio transversal descriptivo con análisis exploratorio. La población del estudio consistió en los integrantes del Centro de Adultos Mayores (CAMChiclayo) perteneciente a EsSalud, con un total de 350 adultos mayores, que acuden al menos una vez por semana. Se excluyó aquellos con antecedente de trastorno tiroideo, depresivo y/o cognitivo y consumo de anticoagulantes y/o antiagregantes; además, el no saber leer y escribir. Se consideró adulto mayor a aquella persona con edad mayor o igual a 60 años, definición dada por la OMS. El muestreo fue no probabilístico-consecutivo. El tamaño muestral en base a una población de 350, nivel de confianza de $95 \%$, frecuencia esperada de hipotiroidismo subclínico de 9,4\% (12) y precisión de $5 \%$ fue de 96 adultos mayores. Se dio una charla informativa previa acerca de la importancia del tamizaje tiroideo, sobre hipotiroidismo subclínico y sus posibles consecuencias en la salud mental.
En el mismo día se les tomó una muestra de sangre de $5 \mathrm{~mL}$ para dosaje de TSHus y T4 libre y los tests para diagnóstico de depresión y deterioro cognitivo. Las tomas de muestra se hicieron en noviembre de 2012 y febrero de 2014, en el mismo laboratorio.

A todos los pacientes se les cuantificó la tirotropina sérica (TSH), mediante la técnica de enzyme-linked immunosorbent assay (ELISA) tipo sándwich; se utilizó el reactivo Accubind que considera valores normales de TSH de 0,3 a 4,2 $\mu \mathrm{U} /$ $\mathrm{mL}^{(13)}$; se consideró un valor anormal por encima de 4,5, según la Guía de práctica clínica de Corrales ${ }^{(14)}$. Las muestras fueron procesadas en el fotómetro de marca ChemWell-T (instrumentos utilizados en el laboratorio particular). Los valores de tiroxina libre (T4l) fueron medidos mediante ELISA ${ }^{(13)}$; el valor de referencia normal es de 12,0 a 22,0 $\mu \mathrm{U} / \mathrm{mL}^{(14)}$. Las muestras de sangre no fueron utilizadas para fines ajenos al estudio, siendo descartadas luego del dosaje hormonal. Se recolectó datos demográficos y antecedentes importantes, como la presencia de diabetes mellitus, hipertensión arterial y dislipidemia.

Para valorar deterioro cognitivo se utilizó el test de tamizaje cognitivoconductual (MMSE, mini-mental state examination) ${ }^{(15,16)}$, validado y utilizado en grupos comunitarios en la práctica y la investigación clínica ${ }^{(17)}$ y en las primeras etapas de los estudios epidemiológicos ${ }^{(15)}$; evalúa cinco áreas: orientación en tiempo y espacio, registro, atención y cálculo, evocación y lenguaje, para personas con más de 2 años de instrucción, que sepan leer y escribir. Los puntos de corte para deterioro cognitivo son menos de 24 y más de 11. En el Perú se modificó y validó la aplicación del MMSE en 1999, para la encuesta de la OPS 'Salud, Bienestar y Envejecimiento' (18), con sensibilidad de $79,8 \%$, especificidad de $81,3 \%$, valor predictivo positivo de $86,3 \%$, valor predictivo negativo de $73,0 \%{ }^{(15)}$ y el alfa de Cronbach de 0,81 ${ }^{(16)}$.

Para evaluar depresión, se utilizó la Escala de Depresión Geriátrica de Yesavage Reducida, test autoadminis- trado o heteroadministrado ${ }^{(19)}$, con sensibilidad 80 a $90 \%$, especificidad 70 a $80 \%$ para el punto de corte $\geq 5^{(20)}$.

El proyecto fue aprobado por el Comité de Ética Institucional de la Facultad de Medicina de la Universidad Católica Santo Toribio de Mogrovejo, de Chiclayo, y las autoridades encargadas del CAM-Chiclayo-Lambayeque. Se entregó un consentimiento informado en el que se detalló la gratuidad y confidencialidad del estudio y la posibilidad de retiro del estudio cuando lo deseasen. Se les explicó los beneficios y probables complicaciones. Los adultos mayores con alteraciones en los valores de $\mathrm{TSH}$ y/o T4 libre fueron evaluados por un médico internista, quién brindó consejería y prescribió reemplazo tiroideo a los que lo ameritaban. Se les aconsejó visitar el consultorio de geriatría y/o endocrinología de EsSalud para posterior evaluación y control. Se usó Microsoft Excel V. 2007 y STATA versión 11. Se hizo un análisis descriptivo, con frecuencias y porcentajes y un análisis exploratorio para obtener razones de prevalencia con IC: 95\%, considerando un nivel de significancia de $5 \%$.

\section{RESULTADOS}

Se obtuvo los datos de 84 adultos mayores. Del total, $77 \%$ eran mujeres y $23 \%$ hombres. La edad promedio fue 71,4 \pm 5 años, rango 66 a 82; promedio de edad en hombres $71,94 \pm 4,93$ y en mujeres $71,24 \pm 5,1$. Se encontró que $14,3 \%$ $(12 / 84)$ era soltero, $45,2 \%(38 / 84)$ casados, $29,8 \%$ (25/84) viudos y $10,7 \%$ (9/84) divorciados. Según el grado de instrucción, 17,8\% (15/84) tenía grado de instrucción primaria, $26,2 \%$ (22/84) secundaria y $55,9 \%(47 / 84) \mathrm{su}$ perior (técnico o universitario); 38,1\% (32/84) tenía hipertensión arterial, $19,1 \%$ (16/84) diabetes mellitus, $17,9 \%$ (15/84) diabetes mellitus e hipertensión arterial, 23,8\% (20/84) dislipidemia, y 9,5\% (8/84) diabetes mellitus, hipertensión arterial y dislipidemia al mismo tiempo.

El promedio de TSH y tiroxina libre fue $3,58 \mathrm{mIU} / \mathrm{L} \pm 5,46$ y $15,47 \mathrm{mIU} /$ 
$\mathrm{L} \pm 3,17$, respectivamente. Hubo $13,1 \%$ (11/84) con hipotiroidismo subclínico: $10,7 \%$ mujeres $(9 / 84)$ y $2,4 \%(2 / 84)$ hombres. Adicionalmente, se encontró $4,8 \%$ (4/84) adultos mayores con hipotiroidismo establecido, 2,4\% hipotiroidismo subclínico y ningún participante con hipertiroidismo. De los 11 pacientes con hipotiroidismo subclínico, 3 (27,3\%) tuvieron un TSHus mayor de $10 \mathrm{uU} / \mathrm{mL}$. El promedio del test de Yesavage fue $3,39 \pm 3,4$, rango 0 a 10 . La frecuencia de depresión fue 42,9\% (36/84), 46,1\% (30/65) mujeres y $31,5 \%(6 / 19)$ hombres $(p=0,43)$; no hubo diferencias en la edad entre los deprimidos y los que no lo fueron $(\mathrm{p}=0,2)$.

El promedio del test mini mental fue $27,33 \pm 3,1$, rango 14 a 30 ; la frecuencia de deterioro cognitivo fue $13,1 \%$, $13,8 \%(9 / 65)$ en mujeres y $10,2 \%(2 / 19)$ en hombres $(p=1,00)$; no hubo diferencias en la edad entre los que tuvieron déficit cognitivo y los que no lo tuvieron $(p=0,65)$. De los 11 adultos mayores que mostraron hipotiroidismo subclínico, 27,3\% (3/11) presentaron deterioro cognitivo, $36,4 \%$ (4/11) depresión y ninguno los dos trastornos simultáneamente. Hubo dos pacientes con diabetes e hipotiroidismo subclínico.

De los 36 adultos mayores que presentaban depresión, $11,1 \%$ (4/36) tenía hipotiroidismo subclínico y 2,8\% (1/36) hipotiroidismo establecido; de los 11 con deterioro cognitivo, $27,3 \%(3 / 11)$ presentaba hipotiroidismo subclínico y ninguno con hipotiroidismo manifiesto. Hubo 4 (4,8\%) adultos mayores con depresión y deterioro cognitivo.

Se exploró una razón de prevalencia (RP) entre hipotiroidismo subclínico y depresión, encontrando una RP: 0,85, IC95\% 0,39 a 1,$85 ; p=0,677$, e hipotiroidismo subclínico y deterioro cognitivo RP: 2,48, IC95\% 0,77 a 7,98; $\mathrm{p}=0,134$.

\section{DISCUSIÓN}

La frecuencia de hipotiroidismo subclínico hallada fue mayor a la encontrada en la literatura. Tonial, en Brasil, en un estudio multicéntrico trasversal, en 2007, en 6 casas de reposo en mayores de 60 años encontró una prevalencia de HSC de 7,33\% ${ }^{(21)}$. Llama la atención la mayor frecuencia en nuestro estudio. Esto nos lleva a plantear probablemente una mayor prevalencia en nuestra región. En un estudio poblacional en São Paulo, en 2011, Benseñor halló una prevalencia de 6,5\%: 6,7\% en mujeres y $6,1 \%$ en varones ${ }^{(22)}$. Los resultados pueden diferir, ya que nuestro estudio se hizo en población selecta que acudía a un CAM y el anterior en población general. En el Perú, Campos en un estudio poblacional, en 1995, halló una prevalencia de $9,4 \%$ de hipotiroidismo subclínico ${ }^{(12)}$. Este es el primer estudio regional acerca del problema.

La depresión en el adulto mayor es frecuentemente subdiagnosticada, debido a que algunos de sus aspectos son considerados como parte del proceso de envejecimiento. Juárez, en 2012, en un asentamiento humano en Lima-Perú halló una prevalencia de depresión en adultos mayores de 51,6\%, utilizando el test de Yesavage ${ }^{(23)}$, cifra superior a la de este estudio. Sin embargo, nuestro estudio fue realizado en un centro geriátrico, la población fue diferente y el tamaño muestral menor. Von Muhlenbrock, en un estudio descriptivo, realizado en 2011 en adultos mayores con menos de 24 horas de ingreso al Hospital militar de Santiago ${ }^{(24)}$, utilizando el mismo test encontró una frecuencia de 22,13\% de depresión, siendo más frecuente en mujeres $(28,12 \%, p=0,129)$ $y$ en viudos $(38,46 \%, p=0,035)^{(24)}$. Esta cifra es menor a la de nuestro estudio, posiblemente porque la depresión sería más frecuente en nuestro país que en Chile.

La frecuencia de deterioro cognitivo de este estudio es mucho menor a la encontrada por Chávez en adultos mayores con enfermedad cardiovascular, de consulta externa del Hospital Almanzor Aguinaga Asenjo (39,7\%); las diferencias podrían deberse al mayor riesgo de deterioro cognitivo en paciente cardiovasculares y que acuden por propia iniciativa a consulta ${ }^{(14)}$. En el Hospital
Cayetano Heredia, en Lima, en un análisis secundario del estudio "Valoración geriátrica integral en adultos mayores hospitalizados a nivel nacional", se halló una frecuencia de deterioro cognitivo leve de $24 \%$ y moderado de $12 \%$. Sin embargo, en este estudio se utilizó el test de Pfeiffer, que considera categorías para el DC; además, fue en pacientes hospitalizados ${ }^{(25)}$.

El tamizaje de deterioro cognitivo en adultos mayores es importante, pues su presencia se asocia a mayor mortalidad (26).

No se halló asociación entre hipotiroidismo subclínico y depresión. Chueire ${ }^{(27)}$, en un estudio trasversal descriptivo, al estudiar 323 adultos mayores halló que los pacientes con hipotiroidismo subclínico tuvieron 4 veces la probabilidad de sufrir de depresión $(\mathrm{OR}=4,886$; IC95\% 2,768 a 8,627) (27). Almeida, en Brasil, en 2007, en un estudio trasversal de pacientes provenientes de consulta externa halló que los pacientes con hipotiroidismo subclínico tenían 2,3 veces la probabilidad de tener depresión en comparación a los eutiroideos. (87\% versus $60,5 \%$, $\mathrm{p}<0,001)^{(28)}$. Los resultados de nuestro estudio son diferentes, probablemente por el uso de un instrumento diferente (test de Beck fue en el de Almeida y los criterios del DSMIV en el de Chueire); además, ambos estudios fueron desarrollados en poblaciones diferentes.

Tampoco se halló asociación entre hipotiroidismo subclínico y deterioro cognitivo. Wijsman en un estudio multicéntrico, prospectivo y longitudinal halló que los pacientes con hipotiroidismo subclínico, al seguimiento no tuvieron mayor riesgo de presentar deterioro cognitivo ${ }^{(8)}$. Parsaik, en un estudio analítico transversal de base poblacional, en Minnesota, no halló asociación entre hipotiroidismo subclínico y deterioro cognitivo: RP 0,88 $\left[0,38\right.$ a 2,03] ${ }^{(29)}$.

En adultos mayores con hipotiroidismo subclínico y TSHus $>10 \mathrm{uU} / \mathrm{L}$ existe un mayor riesgo de desarrollar hipotiroidismo establecido, en el tiem- 
po ${ }^{(30)} ; 3(27,3 \%)$ de nuestros pacientes tenían esta característica.

Para el caso de depresión y/o deterioro cognitivo, es recomendable que el CAM trabaje en apoyo de un profesional de la salud mental para el diagnóstico, manejo y control posterior. Un diagnóstico precoz retrasa la progresión de la enfermedad y mejora la calidad de vida de los pacientes y el de sus familiares.

Entre las limitaciones del estudio, es importante resaltar el tipo de muestreo, el cual fue no probabilístico-consecutivo, y el no haber alcanzado el tamaño muestral calculado (84/96). La población que acude a los centros de adulto mayor son personas en su mayoría controladas, con evaluaciones periódicas por médico general y psicóloga, no siendo representativos de la población general. Otra limitación fue la toma de muestra realizada en dos periodos diferentes. Si bien es cierto un internista evaluó en una oportunidad a la mayoría de adultos mayores con hipotiroidismo subclínico, el seguimiento de los mismos ha sido solamente por el médico del CAM.

El uso del test de Yesavage -recomendado y útil en adultos mayores- y ser el primer estudio que evalúe estos aspectos en Lambayeque, son una fortaleza.

En conclusión, existe una elevada frecuencia de hipotiroidismo subclínico, depresión, y deterioro cognitivo en este Centro de Adultos Mayores de Lambayeque. No se halló asociación entre hipotiroidismo subclínico y depresión, ni hipotiroidismo subclínico con deterioro cognitivo.

\section{AGRADECIMIENTOS}

Se agradece al Centro de Adultos Mayores-Chiclayo por abrirnos las puertas a la investigación, así como obtener datos base para estudios posteriores.

\section{REFERENCIAS BIBLIOGRÁFICAS}

1. Iglesias P, Lázaro J, Velasco Gy Diez J. Disfunción tiroidea en población laboral hospitalaria. Rev Clín
Española. 2010;210(10):505-8.

2. Zárate A, Hernández A, Basurto L, Saucedo R. La enfermedad tiroidea es más frecuente en la mujer. Acta Médica Grupo Ángeles. 2010;8(2).

3. Martínez L. frecuencia de hiper e hipotiroidismo subclinico en pacientes derivados al Instituto de Investigaciones en ciencias de la Salud. Mem Inst Investig Cienc Salud. Paraguay. 2008. Fecha de acceso 20 de febrero del 2014. Disponible en: http:// scielo.iics.una.py/pdf/iics/v6n2/v6n2a05.pdf.

4. Cooper D, Biondi B. Subclinical thyroid disease. Lancet. 2012 Mar;379(9821):1142-54. doi: 10.1016/ S0140-6736(11)60276-6.

5. Rodondi N, den Elzen WP, Bauer DC, Cappola AR, Razvi S, Walsh JP, et al; Thyroid Studies Collaboration. Subclinical hypothyroidism and the risk of coronary heart disease and mortality. JAMA. 2010; Sep 22;304(12):1365-74. doi: 10.1001/ jama.2010.1361.

6. Gencer B, Collet T, Virgini V, Bauer D, Gussekloo J, Cappola A, et al; Thyroid Studies Collaboration. Subclinical thyroid dysfunction and the risk of heart failure events: an individual participant data analysis from 6 prospective cohorts. Circulation. 2012 Aug 28;126(9):1040-9.

7. John JA, Henderson VW, Gatto NM, McCleary CA, Spencer CA, Hodis HN, Mack WJ. Mildly elevated TSH and cognition in middle-aged and older adults. Thyroid. 2009 Feb 19;19(2):111-7. doi: 10.1089/ thy.2008.0226.

8. Wijsman L, Craen A, Trompet S, Gussekloo J, Stott D, Rodondi N, et al. Subclinical thyroid dysfunction and cognitive decline in old age. Plos One. 2013;8(3):e59199. doi: 10.1371/journal. pone.0059199.

9. Bacca A, González A, Uribe A. Validación de la escala de depresión de Yesavage (versión reducida) en adultos mayores Colombianos. Red de Revistas Cientificas de América Latina, el Caribe, España y Portugal. 2005;1(4):53-63.

10. OMS. Depresión. Disponible en: http://www.who. int/topics/depression/es/

11. Delgado C, Salinas P. evaluación de las alteraciones cognoscitivas en adultos mayores. Rev Hosp Clin Univ de Chile. 2009;20:244-51.

12. Campos M, Casado T, Solis J. Hipotiroidismo oculto en adultos mayores de Lima-Perú. Rev Med Her. 1995;6(1).

13. Accubind. Thryrotropin (TSH) Test System. Disponible en: www.monobind.com

14. Corrales J, Galofré J, Pérez B, Cantón A, Alonso N, Pérez A y col. Guía clínica del diagnóstico y tratamiento de la disfunción tiroidea subclínica. Endocrinol Nutr. 2007;54(1):44-52.

15. Chávez L, Núñez I, Díaz C, Poma J. Tamizaje de deterioro cognitivo leve en adultos mayores con enfermedad cardiovascular en un Hospital Nacional de Chiclayo, Perú. Rev Méd Risaralda. 2013 Dic;19(3).

16. Petersen C, Stevens J, Ganguli M, Tangalos E, Cummings J, DeKosky S. Practice Parameter: early detection of dementia: mild cognitive impairment (an evidence - based review) Report of the Quality Standards Subcommittee of the American Academy of Neurology. Neurology. 2001;56:1133-42.

17. Ávila M, Roca A, Blanco K. Manejo en la atención primaria de personas con deterioro cognitivo. Cognitive Impairment Management in the Primary Care. Correo Científico Médico de Holguín. 2007;11.

18. Organización Panamericana de la Salud / Organización Mundial de la Salud. Guía clínica para atención primaria a las personas adultas mayores. Modulo 4: Evaluación del estado mental y emo- cional del adulto mayor. Oficina Regional de la Organización Mundial de la Salud; 2002.

19. D'Art P, Katona P, Mullan E. Screening, detection and management of depression in elderly primary care attenders: The acceptability and perfomance of the 15-item geriatric depression scale (GDS 15) and the development of shorter versions. Fam Pract. 1994;11:260-6.

20. Hoyl T. Development and testing of a five-item version of the Geriatric Depression Scale. J Am Geriatr Soc. 1999;47:873-8.

21. Tonial, R, Rosa L, Da I. Francini B, Sonego A, et al. Prevalência de hipotiroidismo em residentes das seis instituições para idosos do municipio de Criciúma. ACM arq. catarin. Med. Brazil. 2007;36(4):37-41.

22. Benseñor I, Carvalho A, Lotufo $P$, Menezes $P$, Scazufca M, et al. Prevalence of thyroid disorders among older people: results from the Sao Paulo Ageing \& Health Study. Cad. saúde pública. 2011;27(1):155-61.

23. Juárez J, León A, Alata V. Evaluación del grado de depresión de adultos mayores de 60 años del AA.HH “Viña Alta”- La Molina, Lima-Perú. Rev Horiz Med. 2012;12(2):26-9.

24. Von Muhlenbrock F, Gómez R, González M, Rojas A, Vargas, Mühlenbrock C. Prevalencia de depresión en pacientes mayores de 60 años hospitalizados en el servicio de Medicina interna del Hospital Miitar de Santiago. Rev Chil NeuroPsiquiat. 2011;49(4):331-7.

25. Varela L. Características del deterioro cognitivo en el adulto mayor hospitalizado a nivel nacional. Rev Soc Per Med Inter. 2004;17(2):37-42.

26. Kelman H, Thomas C, Kenndy J, Cheng J. Cognitive impairment and mortality in older community residents. Am J Public Health. 1994;84:1255-60.

27. Chueire V, Romaldini J, Ward L. Subclinical hypothyroidism increases the risk for depression in the elderly. Arch Gerontol Geriatr. 2007;44(1):21-8.

28. Almeida C. Subclinical hypothyroidism: psychiatric disorders symptoms. Rev Bras Psiquiatr. 2007;29(2):157-9.

29. Parsaik A, Singh B, Roberts R, Pankratz S, Edwards K, Geda Y, et al. Hypothyroidism and risk of mild cognitive impairment in elderly persons: a population-based study. JAMA Neurol. 2014 Feb;71(2):201-7. doi: 10.1001/ jamaneurol.2013.5402.

30. Somwaru L1, Rariy CM, Arnold AM, Cappola AR. The natural history of subclinical hypothyroidism in the elderly: the cardiovascular health study. $J$ Clin Endocrinol Metab. 2012;97(6):1962-9. doi: 10.1210/jc.2011-3047.

Artículo recibido el 13 de marzo de 2014 y aceptado para publicación el 3 de abril de 2014.

Conflictos de interés:

Franco León Jiménez es miembro del Comité de Ética e Investigación de la Universidad Católica Santo Toribio de Mogrovejo, pero no participó en la aprobación de este proyecto.

\section{Financiamiento propio.}

Correspondencia:

Autora: Carol Marianella Gonzales Gonzales

Dirección: Urb. La Florida Mz M lote 28-Chiclayo

Teléfono celular: 971493018

Correo electrónico: caritogg17@hotmail.com 